\title{
DUALITY FOR GROUPS
}

\author{
SAUNDERS MACLANE ${ }^{1}$
}

\section{The PHENOMENON OF DUALITY}

1. Abelian groups. Certain dualities arise in those theorems of group theory which deal, not with the elements of groups, but with subgroups and homomorphisms. For example, a free abelian group $F$ may be characterized in terms of the following diagram of homomorphisms:

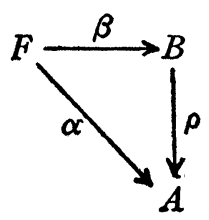

Theorem 1.1. The abelian group $F$ is free if and only if, whenever $\rho: B \rightarrow A$ is a homomorphism of an abelian group $B$ onto an abelian group $A$ and $\alpha: F \rightarrow A$ a homomorphism of $F$ into $A$, there exists $a$ homomorphism $\beta: F \rightarrow B$ with

$$
\rho \beta=\alpha .
$$

If $F$ is known to be free, with generators $g_{i}, \beta$ may be constructed by setting $\beta g_{i}=b_{i}$, with $b_{i}$ so chosen that $\rho b_{i}=\alpha g_{i}$. Conversely, let $F$ have the cited property and represent $F$ as a quotient group $F_{0} / R_{0}$, where $F_{0}$ is a free abelian group. Choose $A=F$ and $B=F_{0}$ in (1.1), let $\alpha$ be the identity, and $\rho$ the given homomorphism of $F_{0}$ onto $F$ with kernel $R_{0}$. Then, by (1.2), $\alpha=\rho \beta$ is an isomorphism, hence $\beta$ has kernel 0 and thus is an isomorphism of $F$ into $F_{0}$. Therefore $F$ is isomorphic to a subgroup of a free group $F_{0}$, so is itself free.

The analogous theorem is true for free nonabelian groups, when $A$ and $B$ are interpreted as arbitrary (not necessarily abelian) groups; the proof uses the Schreier theorem [14 $]^{2}$ that a subgroup of a free group is free.

An abelian group $D$ is said to be infinitely divisible if for each $d \in D$ and each integer $m$ there exists in $D$ an element $x$ such that $m x=d$. Such groups may be characterized by a similar diagram

An address delivered before the Chicago meeting of the Society on November 27, 1948 by invitation of the Committee to Select Hour Speakers for Western Sectional Meetings; received by the editors December 13, 1949.

${ }^{1}$ Essential portions of this paper were developed while the author held a fellowship from the John Simon Guggenheim Memorial Foundation.

${ }^{2}$ Numbers in brackets refer to the bibliography at the end of the paper. 


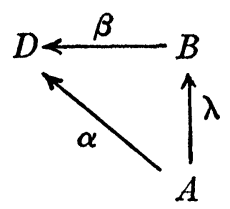

THEOREM 1.1'. The abelian group $D$ is infinitely divisble if and only if, whenever $\lambda: A \rightarrow B$ is an isomorphism of an abelian group $A$ into an abelian group $B$ and $\alpha: A \rightarrow D$ a homomorphism of $A$ into $D$, there exists a homomorphism $\beta: B \rightarrow D$ with

$$
\beta \lambda=\alpha .
$$

Proof. Let $D$ be infinitely divisible. Since $\lambda$ is an isomorphism of $A$ into $B$, the construction of $\beta$ is essentially that of extending a homomorphism $\alpha$ of $A$ into $D$ to a larger group containing $A$; this construction, using suitable transfinite methods, is well known.

Conversely, let $D$ be an abelian group with the property cited in the theorem, and let $d$ be an element in $D$ and $m$ any integer. The cyclic subgroup $Z$ generated by $d$ can be embedded in a cyclic group $B$ generated by an element $b$ in such fashion that $m b=d$. In $\left(1.1^{\prime}\right)$ take $A=Z, \alpha$ the identity, and $\lambda$ the identity injection of $Z$ into $B$. There is then a $\beta$ as in $\left(1.2^{\prime}\right)$; if $\beta b=x$, then $m x=\beta(m b)=\beta \lambda(d)$ $=\alpha(d)=d$. Hence the equation $m x=d$ has a solution in $D$, and $D$ is infinitely divisible.

In this pair of "dual" theorems the hypotheses differ only in the direction of the arrows in the diagrams (1.1) and (1.1') and in the replacement of $\rho$, a "homomorphism onto," by $\lambda$ an "isomorphism into"; the conclusions differ only in the direction of the arrows and in the inversion of the order of factors in the products (1.2) and $\left(1.2^{\prime}\right)$. In this sense free abelian groups ${ }^{3}$ are dual to infinitely divisible abelian groups.

There are other "dual" properties of free and infinitely divisible abelian groups. Any subgroup of a free group is free; any quotient group of an infinitely divisible group is infinitely divisible. Any abelian group is isomorphic to a quotient group of a free abelian group; any abelian group is isomorphic to a subgroup of an infinitely divisible group (that is, can be embedded in an infinitely divisible group). If a free abelian group $F$ is a factor group of an abelian group, it is a direct factor; if an infinitely divisible group $D$ is a subgroup of an abelian group, it is a direct factor.

${ }^{3}$ Call the dual (in this sense) of a free (nonabelian) group a fascist group. R. Baer has shown me a proof of the elegant theorem: every fascist group consists only of the identity element. 
The last property has an application to the characterization of group extensions. Given abelian groups $G$ and $A$, an (abelian) extension of $G$ by $A$ is an abelian group $E$ with $G$ as subgroup and $A$ as corresponding factor group $A=E / G$. More exactly, an extension is a diagram

$$
G \stackrel{\lambda}{\rightarrow} E \stackrel{\rho}{\rightarrow} A
$$

where $\lambda$ is an isomorphism of $G$ into $E, \rho$ a homomorphism of $E$ onto $A$, and the kernel of $\rho$ is the image of $\lambda$. The set of all extensions of $G$ by $A$, with a suitable equivalence relation and a suitable composition, constitutes a group $\operatorname{Ext}(A, G)$. This group has an alternate expression ([7] Theorem 10.1) in terms of any representation of $A$ as a quotient group of a free group.

Theorem 1.2. If $A$ is isomorphic to $F / R$, where $F$ is a free abelian group with subgroup $R$, then

$$
\operatorname{Ext}(A, G) \cong \operatorname{Hom}(R, G) / \operatorname{Hom}(F, G) \mid R,
$$

where Hom $(R, G)$ denotes the group of homomorphisms of the group $R$ into $G$, and Hom $(F, G) \mid R$ the subgroup of those homomorphisms of $R$ into $G$ which can be extended to homomorphisms of $F$ into $G$.

A similar and relatively simple argument will prove a dual theorem. The dual of an extension of $G$ by $A$ is described by a diagram like (1.3) with the arrows reversed and the terms "isomorphism into" and "homomorphism onto" interchanged; thus the dual of an extension of $G$ by $A$ is an extension of $A$ by $G$. The dual theorem now reads

TheOREM 1.2'. If $A$ is isomorphic to a subgroup $S$ of an infinitely divisible group $D$, then

$$
\operatorname{Ext}(G, A) \cong \operatorname{Hom}(G, D / S) / \operatorname{Hom}_{S}(G, D),
$$

where Hom $(G, D / S)$ denotes the group of homomorphisms of $G$ into the factor group $D / S$, and $\operatorname{Hom}_{S}(G, D)$ the subgroup of those homomorphisms of $G$ into $D / S$ which can be obtained by a homomorphism of $G$ into $D$, followed by the canonical projection of $D$ onto $D / S$.

It should be noted that the $\operatorname{subgroups} \operatorname{Hom}(F, G) \mid R$ and $\operatorname{Hom}_{S}(G, D)$ which appear in (1.4) and $\left(1.4^{\prime}\right)$ can be described in strictly dual fashion. Thus in (1.4) let $\kappa: R \rightarrow F$ denote the identity injection of $R$ into $F$. Then a homomorphism $\gamma$ of $R$ into $G$ lies in the subgroup $\operatorname{Hom}(F, G) \mid R$ if and only if there exists a $\beta$,

$$
\beta: F \rightarrow G \text { with } \beta \kappa=\gamma .
$$


Dually, let $\pi: D \rightarrow D / S$ be the canonical projection of the infinitely divisible group $D$ onto the factor group $D / S$. Then a homomorphism $\gamma$ of $G$ into $D / S$ lies in the subgroup $\operatorname{Hom}_{S}(G, D)$ if and only if there exists a $\beta$

$$
\beta: G \rightarrow D \text { with } \pi \beta=\gamma .
$$

2. A table of dualities. The duality under discussion is a process which assigns a dual statement to each of certain statements about groups and homomorphisms. Each homomorphism $\alpha$ is understood to be a homomorphism of a specified group $G$ into a specified group $H$; we write $\alpha: G \rightarrow H$ and call $G$ the domain and $H$ the range of $\alpha$. Note that the range may be larger than the image $\alpha(G)$.

If $S$ is a subgroup of $G$ (notation $S \subset G$ ), then the injection $\kappa$ $=[G \supset S]$ of $S$ into $G$ is that homomorphism of $S$ into $G$ with $\kappa(s)=s$ for every $s \in S$. If $N$ is a normal subgroup of $G$, then $Q=G / N$ is a quotient group of $G$ (notation $Q \leqq G$ ), and the projection $\pi=[Q \leqq G]$ of $G$ onto $Q$ is that homomorphism of $G$ onto $Q$ for which $\pi(g)$ is the coset $g N$ for every $g \in G$. The systematic use of these injection and projection homomorphisms is at the heart of our formulation of the duality phenomena.

We consider any statement $S$ about groups which does not make reference to the elements of the groups involved, but only to homomorphisms with these groups as domains and ranges, to the products of homomorphisms, to subgroups and quotient groups, injection and projection. The statement dual to $S$ is then the statement obtained by carrying out the following interchanges in $S$.

$\begin{array}{ll}\alpha: G \rightarrow H & \alpha: H \rightarrow G \\ \text { domain } \alpha=G & \text { range } \alpha=G \\ \alpha \text { is an isomorphism into } & \alpha \text { is a homomorphism onto } \\ \text { product } \beta \alpha & \text { product } \alpha \beta \\ S \text { is a subgroup of } G & Q \text { is a quotient group of } G \\ \text { the injection }[G \supset S]: S \rightarrow G & \text { the projection }[Q \leqq G]: G \rightarrow Q .\end{array}$

A difficulty appears at once. The relation of inclusion for subgroups is transitive, in that $T \subset S$ and $S \subset G$ imply $T \subset G$. However, if $R \leqq Q$ and $Q \leqq G$, then $R$ cannot be a quotient group of $G$, consisting of cosets of $G$, because $R$ consists of cosets of cosets of $G$. The second isomorphism theorem shows only that $R$ is isomorphic to a quotient group of $G$. By suitably redefining the notion of a quotient group, this difficulty will be removed (cf. $\$ 12$ below).

To apply this duality to nonabelian groups we consider the dual to the statement " $S$ is a normal subgroup of $G$." The usual definition of 
normality refers to elements of the groups, hence cannot be dualized directly. Let us say that a homomorphism $\beta: G \rightarrow H$ is a zero homomorphism (briefly, $\beta=0$ ) if $\beta$ maps all of $G$ into the identity element of $H$. The statement " $\beta=0$ " turns out to be self dual $(\$ 15)$. If $S$ and $S^{\prime}$ are subgroups of $G,[G \supset S]$ and $\left[G \supset S^{\prime}\right]$ the corresponding injections into $G$, we say that $S$ dominates $S^{\prime}$ in $G$ if and only if, for every homomorphism $\alpha: G \rightarrow H$,

$$
\alpha[G \supset S]=0 \text { implies } \alpha\left[G \supset S^{\prime}\right]=0 .
$$

It then appears that a subgroup $S$ is normal in $G$ if and only if $S$ contains every subgroup $S^{\prime}$ of $G$ which it dominates. This characterization can be dualized to define a conormal quotient group of $G$; it then appears that every quotient group is conormal in this sense!

This illustrates the fact that the dual of a true statement about groups need not be true. Another more familiar example is the following. Every subgroup $S$ of a quotient group $G / N$ of $G$ is a quotient group of a subgroup of $G$; indeed $S=M / N$, for a suitable subgroup $M$, with $G \supset M \supset N$. However, a quotient group $S / N$ of a subgroup $S$ of $G$ need not be a subgroup of a quotient group of $G$, because $N$, though normal in the subgroup $S$, need not be normal in $G$. Another example is the Kurosch-Birkhoff-Jordan-Hölder theorem for transfinite ascending sequences $[5$, p. 89]; the dual is not true, even for abelian groups.

It is nevertheless true that the duals of a large class of true statements about groups are true, and it is our objective to delimit this class of statements.

3. Free products and direct products. Let $A \times B$ be the direct (or cartesian) product of the groups $A$ and $B$, defined as the group of pairs $(a, b)$ for $a \in A, b \in B$. Let $\alpha$ and $\beta$ denote the natural homomorphisms $\alpha(a, b)=a, \beta(a, b)=b$ of the direct product onto its respective factors. The direct product may then be described conceptually in terms of $\alpha$ and $\beta$ and the diagram

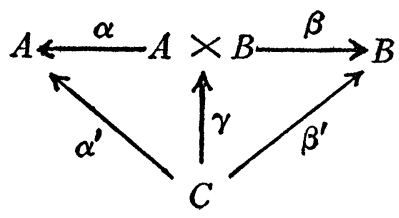

as follows. Given any group $C$, and any homomorphisms $\alpha^{\prime}$ and $\beta^{\prime}$ of $C$ into $A$ and $B$ respectively, there exists one and only one homomorphism $\gamma: C \rightarrow A \times B$ with

$$
\alpha \gamma=\alpha^{\prime}, \quad \beta \gamma=\beta^{\prime} .
$$


Specifically, $\gamma(c)=\left(\alpha^{\prime} c, \beta^{\prime} c\right)$ is such a homomorphism, and is clearly the only such. This property of the diagram (3.1) determines the direct product $A \times B$ and its mappings $\alpha$ and $\beta$ up to an isomorphism; hence it may serve as a definition of the direct product.

Let $A * B$ be the free product of the groups $A$ and $B$, defined in the usual fashion (cf. $[18$, p. $45 ; 1 ; 21]$ ) as the set of all words $a_{1} b_{1} a_{2} b_{2} \ldots$ $a_{n} b_{n}$ for $a_{i} \in A, b_{i} \in B$ with multiplication defined by juxtaposition of words, equality by the process of removing any $b_{i}$ (or any $a_{i}$ ) equal to 1 , and multiplying the two adjacent $a^{\prime}$ 's, together with the inverse and iterations of this process. With this free product we associate the isomorphism $\alpha$ of the first factor $A$ into $A * B$, obtained by mapping each $a$ in $A$ into the word " $a$," and the analogous isomorphism $\beta: B \rightarrow A * B$. The free product may then be described conceptually by the diagram

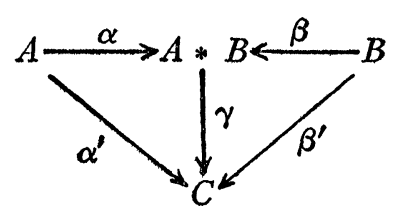

as follows. Given any group $C$, and any homomorphisms $\alpha^{\prime}$ and $\beta^{\prime}$ of $A$ and $B$, respectively, into $C$, there exists one and only one homomorphism $\gamma: A * B \rightarrow C$ such that

$$
\gamma \alpha=\alpha^{\prime}, \quad \gamma \beta=\beta^{\prime} .
$$

Specifically, $\gamma\left(a_{1} b_{1} \cdots a_{n} b_{n}\right)=\left(\alpha^{\prime} a_{1}\right)\left(\beta^{\prime} b_{1}\right) \cdots\left(\alpha^{\prime} a_{n}\right)\left(\beta^{\prime} b_{n}\right)$ is such a homomorphism, and is the only one. Again, this description determines the free product, together with its mappings $\alpha$ and $\beta$, up to an isomorphism.

The diagram (3.1) is dual to $\left(3.1^{\prime}\right)$, while the product relations (3.2) are dual to $\left(3.2^{\prime}\right)$. The mappings $\alpha$ and $\beta$ of (3.1) are homomorphisms onto; in $\left(3.1^{\prime}\right)$ they are isomorphisms into. If the intersection of the kernels of $\alpha^{\prime}$ and $\beta^{\prime}$ in (3.1) is the unit group, then $\gamma$ in (3.1) is an isomorphism into. If the union of the images of $\alpha^{\prime}$ and $\beta^{\prime}$ in $\left(3.1^{\prime}\right)$ is the group $C$, then $\gamma$ in $\left(3.1^{\prime}\right)$ is a homomorphism onto. These two statements can be so reformulated as to be strictly dual in our sense (the notion of "kernel" must be replaced by a suitable notion of "coimage," as described in $\$ 16$ below.)

The proof of the existence of the direct product is not dual to the proof of the existence of the free product, for both proofs involve reference to the elements of the groups concerned. However, the proof that the direct product is unique up to an isomorphism can be phrased so as to be exactly dual to the proof of the uniqueness of the 
free product up to an isomorphism. Similarly, the proofs of the associative and commutative laws for direct products, formulated in terms of diagrams like (3.1), are dual to the proofs of the corresponding laws for the free product, as will be indicated again in $\$ 18$ below.

4. Composition series and chief series. A composition series for the (finite) group $G$ is a sequence of subgroups

$$
G=M_{0} \supset M_{1} \supset M_{2} \supset \cdots \supset M_{k-1} \supset M_{k}=(1),
$$

such that each subgroup $M_{i}$ is a maximal proper normal subgroup of the group $M_{i-1}$, for $i=1, \cdots, k$. A chief series for the finite group $G$ is a sequence of normal subgroups of $G$,

$$
G=N_{m} \supset N_{m-1} \supset N_{m-2} \supset \cdots \supset N_{1} \supset N_{0}=(1),
$$

such that each $N_{i}$ is maximal among the proper subgroups of $N_{i+1}$ normal in $G$. This description is not a dual of the preceding description, but consider instead the quotient groups $Q_{i}=G / N_{i}$. Then the chief series becomes a sequence of quotient groups of $G$,

$$
G=Q_{0} \geqq Q_{1} \geqq Q_{2} \geqq \cdots \geqq Q_{m-1} \geqq Q_{m}=\left(1^{\prime}\right),
$$

with each $Q_{i}$ a maximal proper quotient group of $Q_{i-1}$, for $i=1, \cdots$, $m$. Conversely, any such sequence of quotient groups of $G$ determines uniquely the corresponding chief series (4.2). The description of $\left(4.1^{\prime}\right)$ is dual to that of (4.1), because the maximal proper quotient groups may be described equivalently as maximal proper conormal quotient groups, with our previous definition of conormality. The last term of the series $\left(4.1^{\prime}\right)$ is the unit quotient group ( $\left.1^{\prime}\right)$ $=G / G$ of $G$, which can be described as that quotient group of $G$ which is a quotient of every quotient group of $G$. This is dual to a description of the unit subgroup (1) of $G$.

We next dualize the formula $G / M$. The quotient group $Q=G / M$, for $M$ normal in $G$, can be characterized in terms of injections and projections by the properties

(i) $[G / M \leqq G][G \supset M]=0$;

(ii) If $[R \leqq G][G \supset M]=0$, then $R \leqq G / M$.

Dually, each conormal quotient group $Q$ of $G$ determines a corresponding normal subgroup of $G$, denoted by $G \div Q$ and characterized by the dual properties

(i') $[Q \leqq G][G \supset(G \div Q)]=0$;

(ii') If $[Q \leqq G][G \supset S]=0$, then $S \subset(G \div Q)$, which state in effect that $G \div Q$ is the kernel of the projection of $G$ on $Q$. Hence, in ordinary terms, 


$$
G \div(G / M)=M \quad G /(G \div Q)=Q,
$$

for $M$ a normal subgroup, $Q$ a conormal quotient group of $G$.

The composition series (4.1) determines a set of quotient groups $M_{i} / M_{i+1}$, for $i=0, \cdots, k-1$. The chief series in the dual form (4.1') determines a set of groups $Q_{i} \div Q_{i+1}$. Since $Q_{i}=G / N_{i}, Q_{i+1}$ $=G / N_{i+1}$, the symbol $Q_{i} \div Q_{i+1}$ designates the kernel of the projection of $Q_{i}$ onto $Q_{i+1}$, and this kernel is exactly the group $N_{i+1} / N_{i}$. Hence the set of quotient groups of the composition series (4.1) corresponds dually to the set of quotients $N_{i+1} / N_{i}$ of the chief series (4.2). The Jordan-Hölder theorem asserting the uniqueness of the set of these quotient groups for a finite group $G$ thus becomes the dual of the Jordan-Hölder theorem for the chief series.

5. Ascending and descending central series. It has long been recognized that the ascending and the descending central series of a group $G$ (cf. $[12 ; 22$, chap. IV]) are dual concepts. We may show that they can be described in strictly dual fashion, in our sense, relative to the group of inner automorphisms of $G$. For any element $a$ in $G$ we denote by $\phi=\phi_{a}$ the corresponding inner automorphism of $G$, with $\phi(g)$ $=a g a^{-1}$ for all $g \in G$, and by $I(G)$ the group of all these inner automorphisms. If $S$ is a subgroup of $G$, then $\phi(S)$ denotes the image of $S$ under $\phi$.

The ascending central series $G \supset \cdots \supset Z_{2} \supset Z_{1} \supset Z_{0}$ for a group $G$ consists of $Z_{0}$, the identity subgroup, $Z_{1}$, the center of $G$, and $Z_{n}$, defined inductively as that normal subgroup of $G$ such that $Z_{n} / Z_{n-1}$ is the center of $G / Z_{n-1}$. In particular, the center $Z_{1}$ consists of all elements of $G$ left fixed by every inner automorphism of $G$. Thus if $S$ is a normal subgroup of $G$ such that

$$
\phi(S)=S \text {, and } \phi \text { induces the identity automorphism on } S,
$$

for every $\phi \in I(G)$, then $S \subset Z_{1}$. Since $Z_{1}$ itself has the property (5.1), it may be characterized as the maximal normal subgroup $S$ of $G$ with this property. This description suggests the following readily proved characterization of the groups $M_{n}=Z_{n}$ of the ascending central series.

THEOREM 5.1. If $I(G)$ is the group of inner automorphisms of $G$, then there exists a unique sequence $G \supset \ldots \supset M_{n} \supset \ldots \supset M_{1} \supset M_{0}$ of normal subgroups of $G$ such that $M_{0}$ is the identity subgroup and, for each $n>0, M_{n}$ is the maximal subgroup $N$ of $G$ with the properties

(i) $N \supset M_{n-1}, N$ normal in $G$;

(ii) For each $\phi$ in $I(G), \phi(N)=N$, and $\phi$ induces the identity automorphism on $N / M_{n-1}$. 
To formulate the dual of (ii), we must use our duality table to interpret the "image" $\phi(N)$ and the "induced" automorphism. Let $\alpha$ be any automorphism of $G$. If $S$ is any subgroup of $G$ and $[G \supset S]$ the corresponding injection of $S$ into $G$, then the product $\alpha[G \supset S]$ is an isomorphism of $S$ onto some subgroup $T$ of $G$, hence may be written uniquely in the form

$$
\alpha[G \supset S]=[G \supset T] \alpha_{S},
$$

where $\alpha_{S}$ is an isomorphism. In this decomposition we may define the group $T$ to be the image of $S$ under $\alpha$, and the isomorphism $\alpha_{S}$ : $S \rightarrow T$ to be the isomorphism induced by $\alpha$ on $S$, in agreement with the usual meaning of an "induced" isomorphism. Dually, let $Q$ be a quotient group of $G$, and $[Q \leqq G]$ the corresponding projection. The product $[Q \leqq G] \alpha$ is then a homomorphism of $G$ onto $Q$, hence may be written uniquely in the form

$$
[Q \leqq G] \alpha=\alpha_{Q}[R \leqq G]
$$

where $R$ is a quotient group of $G$, and $\alpha_{Q}$ an isomorphism of $R$ onto $Q$. We define $R$ to be the coimage of $Q$ under $\alpha$ (in symbols, $R=\phi^{\prime}(Q)$ ), and $\alpha_{Q}: R \rightarrow Q$ to be the isomorphism induced by $\alpha$ on the coimage. In these terms, condition (ii) of Theorem 5.1 becomes the requirement that for each $\phi$ there exists an isomorphism $\phi_{N}: N \rightarrow N$ such that

The dual of Theorem 5.1 may now be formulated as follows.

THEOREM 5.1'. If $I(G)$ is the group of inner automorphisms of $G$, then there exists a unique sequence of (conormal) quotient groups $G \geqq \cdots \geqq Q_{n} \geqq \cdots \geqq Q_{1} \geqq Q_{0}$ of $G$ such that $Q_{0}=G / G$ is the identity quotient group and, for each $n>0, Q_{n}$ is the maximal quotient group $R$ of $G$ with the properties:

(i') $R \geqq Q_{n-1}, R$ conormal in $G$;

(ii') For each $\phi \in I(G), \phi^{\prime}(R)=R$, and $\phi$ induces the identity automorphism on $R \div Q_{n-1}$.

Here again the condition (ii') means that for each $\phi$ there exists an isomorphism $\phi_{R}: R \rightarrow R$ such that

$$
\begin{aligned}
{[R \leqq G] \phi } & =\phi_{R}[R \leqq G], \\
\phi_{R}\left[R \supset\left(R \div Q_{n-1}\right)\right] & =\left[R \supset\left(R \div Q_{n-1}\right)\right] .
\end{aligned}
$$

By setting $Q_{n}=G / L_{n}$, this theorem may be translated into a theorem about a descending chain of normal subgroups $L_{n}$ of $G$. One readily shows that the theorem is valid with $L_{n}$ the groups of the usual 
descending central series; that is, with $L_{1}=[G, G]$, the subgroup of $G$ generated by all commutators in $G$, and in general with $L_{n}$ $=\left[G, L_{n-1}\right]$, the subgroup generated by all commutators of elements in $G$ with elements in $L_{n-1}$.

We have thus shown that in this formulation the descending central series of $G$ is indeed the dual of the ascending central series. We remark in passing that the theorems above are still valid if the group $I(G)$ of inner automorphisms of $G$ is replaced by any group $B(G)$ of automorphisms of $G$.

It may be noted that our formulation of duality in terms of homomorphisms does not suffice to subsume all known "duality" phenomena. In particular, it does not appear to explain the duality between "verbal" and "marginal" subgroups [13], which is, however, an extension of the above duality between ascending and descending central series.

6. Functional and axiomatic duality. For a topological space the duality between homology and cohomology groups with locally compact abelian coefficient groups can be formulated in terms of character groups. Another formulation is suggested by the axiomatic homology theory of Eilenberg and Steenrod $[9 ; 10]$. In this formulation, the axioms for a homology theory refer not to elements of the (relative) homology groups, but only to certain homomorphisms; the dual statements are exactly the axioms for a cohomology theory. For example, any continuous mapping $\xi: X \rightarrow Y$ of one space into a second induces a mapping in the same direction on the homology groups, and in the reverse direction on the cohomology groups of these spaces. One of our chief objectives is that of providing a background in which the proofs for axiomatic homology theory become exactly dual to those for cohomology theory. ${ }^{4}$

Duality phenomena also appear in the case of vector spaces. Each finite-dimensional vector space $V$ over a field $F$ has a dual or conjugate space $V^{*}$, consisting of all linear functionals on $V$ to $F$, and to each linear transformation $T: V \rightarrow W$ there is a conjugate transformation $T^{*}: W^{*} \rightarrow V^{*}$. The passage from a set of transformations to their conjugates inverts the direction of all transformations, interchanges the order of factors in a product of transformations, and replaces a transformation onto by a one-to-one transformation into, hence provides an explicit realization of the type of duality we have discussed. Much the same remarks apply to locally compact abelian groups, under the formation of character groups.

${ }^{4}$ This consideration was suggested to the author by his study of the manuscript of [10]. 
In these instances there is a process assigning to each object a dual object and to each transformation a dual transformation, so that a "functional" duality is present. Similarly, the duality of (plane) projective geometry may be formulated in two ways: functional, by assigning to each figure its polar reciprocal with respect to a fixed conic; axiomatic, by observing that the axioms for plane projective geometry are invariant under the interchange of "point" with "line."

Even for discrete abelian groups or for discrete (infinite-dimensional) vector spaces, a functional duality does not exist. We aim to provide an axiomatic duality covering such cases.

\section{Bicategories}

7. Categories. The notion of an abstract group arises by consideration of the formal properties of one-to-one transformations of a set onto itself. Similarly, the notion of a category [8] is obtained from the formal properties of the class of all transformations $\xi: X \rightarrow Y$ of any one set into another, or of continuous transformations of one topological space into another, or of homomorphisms of one group into another, and so on. Each transformation $\xi$ is associated with a definite domain $X$ and a definite range $Y$; the product or composite $\eta \xi$ of two such transformations $\xi: X \rightarrow Y$ and $\eta: Y^{\prime} \rightarrow Z$ is to be defined only when $Y=Y^{\prime}$ (range $\xi=$ domain $\eta$ ). With these conventions, one has the following formal properties. ${ }^{5}$

Definition. A category $\mathcal{C}$ is a class of elements $\alpha, \beta, \gamma, \cdots$, called "mappings" for certain pairs of which a product $\alpha \beta \in \mathcal{C}$ is defined, subject to the axioms $\mathrm{C}-0$ to $\mathrm{C}-4$.

C-0. (Equality axiom). If $\alpha=\alpha^{\prime}, \beta=\beta^{\prime}$, and the product $\alpha \beta$ is defined, so is the product $\alpha^{\prime} \beta^{\prime}$, and $\alpha \beta=\alpha^{\prime} \beta^{\prime}$.

$\mathrm{C}-1$. If the products $\alpha \beta$ and $(\alpha \beta) \gamma$ are defined, so is $\beta \gamma$.

$\mathrm{C}-1^{\prime}$. If the products $\beta \gamma$ and $\alpha(\beta \gamma)$ are defined, so is $\alpha \beta$.

C-2. (Associative law). If the products $\alpha \beta$ and $\beta \gamma$ are defined, so are the products $(\alpha \beta) \gamma$ and $\alpha(\beta \gamma)$, and these products are equal.

A mapping $I$ of $\mathcal{C}$ is called an identity of $\mathcal{C}$ if (i) $I I$ is defined; (ii) $I \alpha=\alpha$ whenever $I \alpha$ is defined; (iii) $\beta I=\beta$ whenever $\beta I$ is defined.

C-3. (Existence of domain $I$ and range $I^{\prime}$ ). For each $\alpha \in \mathcal{C}$ there are identities $I$ and $I^{\prime}$ in $\mathcal{C}$ such that $\alpha I$ and $I^{\prime} \alpha$ are defined.

C-4. For given identities $I$ and $I^{\prime}$ the class of all mappings $\alpha$ of $\mathcal{C}$ such that both $\alpha I$ and $I^{\prime} \alpha$ are defined is a set (cf. $\$ 8$ below).

The axioms $\mathrm{C}-1, \mathrm{C}-1^{\prime}$, and $\mathrm{C}-2$ together state that the associative law $(\alpha \beta) \gamma=\alpha(\beta \gamma)$ holds, with all terms defined, whenever both products on the left, or both products on the right, or both products

5 These axioms (with C-4 omitted) are equivalent to those given in [8]. 
$\alpha \beta$ and $\beta \gamma$ are known to be defined.

It is convenient to introduce a class of "objects" $A, B, \cdots$ in oneto-one correspondence $A \leftrightarrow I_{A}$ with the identity mappings $I$ of $\mathcal{C}$. Since the identities $I, I^{\prime}$ described in C-3 are unique, the object $A=D(\alpha)$ such that $\alpha I_{A}$ is defined is called the domain of $\alpha$, and the object $B$ such that $I_{B} \alpha$ is defined is the range $B=R(\alpha)$; we then write $\alpha: A \rightarrow B$. These objects have the following properties.

(i) $\alpha=\beta$ implies $D(\alpha)=D(\beta), R(\alpha)=R(\beta)$;

(ii) $A=B$ if and only if $I_{A}=I_{B}$;

(iii) The product $\alpha \beta$ is defined exactly when $D(\alpha)=R(\beta)$, and then $D(\alpha \beta)=D(\beta), R(\alpha \beta)=R(\alpha)$;

(iv) $D\left(I_{A}\right)=R\left(I_{A}\right)=A$;

(v) $\alpha I_{D(\alpha)}=\alpha=I_{R(\alpha)} \alpha$;

(vi) If $D(\alpha)=R(\beta)$ and $D(\beta)=R(\gamma)$, then $\alpha(\beta \gamma)=(\alpha \beta) \gamma$;

(vii) For given objects $A$ and $B$, the class of all $\alpha$ with $R(\alpha)=B$, $D(\alpha)=A$ is a set.

Conversely, these seven properties could serve as a definition of a "category with objects"; they imply the original axioms (C-0)-(C-4) for the mappings of such a category.

A mapping $\theta$ is an equivalence in $C$ if there are mappings $\phi$ and $\psi$ in $\mathcal{C}$ such that $\theta \phi$ and $\psi \theta$ are defined and are identities. Then $\phi=\psi$ is the unique inverse $\theta^{-1}$ of $\theta$. It is itself an equivalence, and

$$
R\left(\theta^{-1}\right)=D(\theta), \quad D\left(\theta^{-1}\right)=R(\theta), \quad\left(\theta^{-1}\right)^{-1}=\theta .
$$

8. Foundations. We shall use the category $G$ of all groups, in which the objects are all groups, and the mappings are all homomorphisms of one group into another-and similarly the category of all topological spaces and continuous mappings, and so on. The apparently illegitimate totalities of "all" groups and "all" homomorphisms can be justified by using the standard von NeumannBernays-Gödel axiomatics for set theory $[2 ; 11]$ in which both the notions of "class" and "set" appear, the sets being more restricted than the classes. We then understand a group to be a set $G$, with multiplication defined by a suitable set of triples; a homomorphism is likewise described by sets. With the cited axioms for set theory, one can then correctly speak of the class of all groups and of the class of all homomorphisms of one group into another. For this reason, we have described a category as a class and have inserted axiom C-4.

9. Bicategories. The primitive concepts of a category are not sufficient to formulate all the duality phenomena, and in particular do not provide for "subgroups versus quotient groups," or "homomorphisms onto versus isomorphisms into." To extend our formula- 
tion, we axiomatize the terms "injection homomorphism of a subgroup into a larger group" and "projection homomorphism of a group onto a quotient group." We can then define homomorphisms onto and isomorphisms into as "supermaps" and "submaps," respectively.

Definition. A bicategor $y^{6} \mathcal{C}$ is a category with two given subclasses of mappings, the classes of "injections" $(\kappa)$ and "projections" $(\pi)$ subject to the axioms BC-0 to BC-6 below. ${ }^{7}$

$\mathrm{BC}-0$. A mapping equal to an injection (projection) is itself an injection (projection).

BC-1. Every identity of $\mathcal{C}$ is both an injection and a projection.

$\mathrm{BC}-2$. If the product of two injections (projections) is defined, it is an injection (projection).

BC-3. (Canonical decomposition). Every mapping $\alpha$ of the bicategory can be represented uniquely as a product $\alpha=\kappa \theta \pi$, in which $\kappa$ is an injection, $\theta$ an equivalence, and $\pi$ a projection.

Any mapping of the form $\lambda=\boldsymbol{\alpha} \theta$ (that is, any mapping with $\pi$ equal to an identity in the canonical decomposition) is called a submap; any mapping of the form $\rho=\theta \pi$ is called a supermap.

BC-4. If the product of two submaps (supermaps) is defined, it is a submap (supermap).

Any product $\kappa_{1} \pi_{1} \cdots \kappa_{n} \pi_{n}$ of injections $\kappa_{i}$ and projections $\pi_{i}$ is called an idemmap.

$\mathrm{BC}-5$. If two idemmaps have the same range and the same domain, they are equal.

BC-6. For each object $A$, the class of all injections with range $A$ is a set, and the class of all projections with domain $A$ is a set.

The inclusion relations between the various classes of mappings can be represented by the following Hasse diagram.

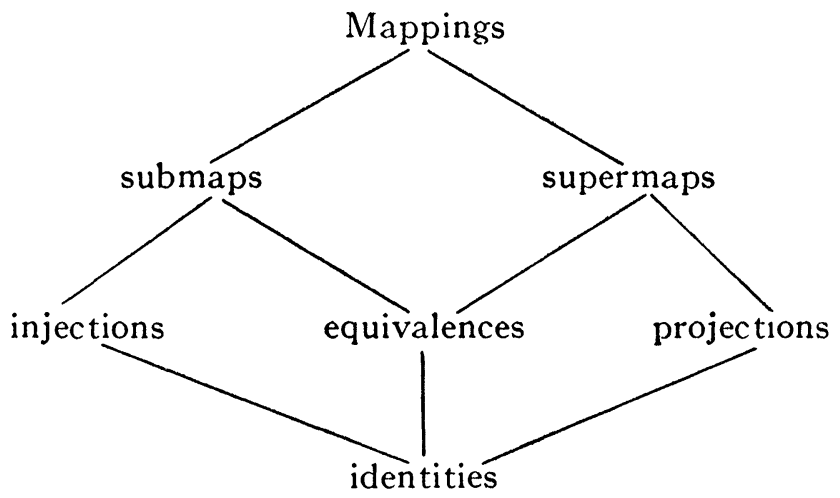

6 The term "bicategory" was suggested by Professor Grace Rose.

7 In the preliminary announcement [16], axiom BC-6 did not appear, and axiom BC-5 was present only in weaker form. 
The axioms show that the intersection of any two such classes is exactly the intersection which may be read off this diagram. Each class of mappings is closed under multiplication (whenever the product is defined). It is convenient to reserve the letters $\lambda, \mu, \nu$ for submaps, $\rho, \sigma, \tau$ for supermaps.

10. The duality principle. The concept of the "dual" of a statement about homomorphisms may now be defined precisely. In a category, the only primitive statements are statements of the forms

$$
\alpha=\beta, \quad \alpha \beta=\gamma ;
$$

We interpret the latter to mean "the product $\alpha \beta$ is defined and is equal to $\gamma$. . All other statements can be expressed in terms of these primitive statements; in particular, we understand the statement " $\alpha \beta$ is defined" to be interpreted as "there exists a $\gamma$ such that $\alpha \beta=\gamma$." A first order statement $S$ in a finite number of letters (which designate mappings of the category in question) is any statement formed from a number of primitive statements of the types (10.1), combined by the standard logical connectives (including quantifiers "for all $\alpha$ " and " $\exists \alpha$ "). The dual of $S$ is the statement obtained from $S$ by the following typographical process: replace each primitive statement $\alpha \beta=\gamma$ by the statement $\beta \alpha=\gamma$, leaving the other primitive statements, all the letters, and all the logical connectives unchanged. The dual is thus obtained by "inverting all products." A similar process will apply to statements which are not of the first order, in that they involve variables for sets of mappings, sets of sets of mappings, and so on.

The dual of any axiom for a category is also an axiom; in particular, $\mathrm{C}-1^{\prime}$ is (except for change in notation) the dual of $\mathrm{C}-1$, and the other axioms are self-dual. A simple metamathematical argument thus proves the

DUALITY PRINCIPLE. If any statement about a category is deducible from the axioms for a category, the dual statement is likewise deducible.

In a bicategory, the only added primitive statements are $\alpha$ is an injection, $\alpha$ is a projection.

The dual of a statement about mappings in a bicategory is now obtained as before, with the added interchange of the terms "injection" and "projection." An inspection of the axioms shows that the duality principle holds for bicategories also.

A statement of group theory may often be formulated in (bi-) categorical form; that is, as a statement about homomorphisms, in- 
jections, projections, identities, and their products. When so formulated, it has a definite dual, but note that there may be several such formulations which lead to essentially different duals. For example, " $Q$ is a quotient group of $G$ " (that is, there is a projection with domain $G$ and range $Q$ ) is equivalent to " $Q$ is a conormal quotient group of $G$." The duals-" $M$ is a subgroup of $G$ " and " $M$ is a normal subgroup of $G$ "-are not equivalent.

11. Partial order in a bicategory. The axioms (especially axiom $\mathrm{BC}-5$ ) suffice to introduce a relation of partial order (under "inclusion") in the objects of a bicategory. We define a mapping $\beta$ to be left cancellable in a category if $\beta \alpha_{1}=\beta \alpha_{2}$ always implies $\alpha_{1}=\alpha_{2}$, and left invertible if $\beta$ has a left inverse $\gamma$, with $\gamma \beta=I_{D(\beta)}$. One may readily prove, in succession, the following results.

LEMMA 11.1. Two injections $\kappa_{1}$ and $\kappa_{2}$ such that $\kappa_{1} \kappa_{2}$ is an identity are themselves identities.

LEMMA 11.2. Every right factor of a submapping is a submapping.

Lemma 11.3. If $\alpha \beta$ is an identity, $\alpha$ is a supermap and $\beta$ a submap.

LEMma 11.4. Every left invertible mapping is a submap, and every submap is left cancellable.

THEOREM 11.5. The class of objects in a bicategory is partially ordered by either of the relations

(11.1) $S \subset B$ if and only if there is an injection $\kappa: S \rightarrow B$;

$\left(11.1^{\prime}\right) Q \leqq A$ if and only if there is a projection $\pi: A \rightarrow Q$.

If $S \subset B$, we call $S$ a subobject of $B$, while if $Q \leqq A, Q$ is a quotientobject of $A$, the terms corresponding to those in group theory. By axiom BC-5 the mappings $\kappa$ and $\pi$ which appear in the dual definitions $(11.1)$ and $\left(11.1^{\prime}\right)$ are unique; it is more suggestive to denote them as

$$
\kappa=[B \supset S]: S \rightarrow B ; \quad \pi=[Q \leqq A]: A \rightarrow Q .
$$

Thus $[B \supset S]$ is a mapping, defined precisely when $S \subset B$ and is then an injection; every injection has this form. The notation is so chosen that

$$
[B \supset S][S \supset T]=[B \supset T],[R \leqq Q][Q \leqq A]=[R \leqq A],
$$

by $\mathrm{BC}-5$, whenever the terms on the left are defined.

In examining prospective examples of bicategories, it is easier to formulate the axioms directly in terms of these constructions on the objects. 
DEFINITION. A bicategory with objects is a category with objects, in the sense of $\S 7$, in which there are mappings $[B \supset S]$ and $[Q \leqq A]$ assigned to certain pairs of objects $B, S$ or $Q, A$, subject to conditions (i)-(vi) below and their duals. Here " $S \subset B$ " means "the mapping $[B \supset S]$ is defined," and dually, while an equation involving the mappings $[B \supset S], \cdots$ is understood to include the assertion that $[B \supset S], \cdots$ is defined. An idemmap is any product $\alpha_{1} \alpha_{2} \cdots \alpha_{m}$, where each $\alpha_{i}$ has one of the forms $[B \supset S]$ or $[Q \leqq A]$. The axioms are

(i) (Equality). $B=B^{\prime}, S=S^{\prime}$, and $S \subset B$ imply $[B \supset S]=\left[B^{\prime} \supset S^{\prime}\right]$.

(ii) For all objects $A,[A \supset A]=I_{A}=[A \leqq A]$.

(iii) Every mapping $\alpha$ has a unique representation

$$
\alpha=[B \supset S] \theta[Q \leqq A], \quad \theta \text { an equivalence. }
$$

(iv) If $\theta: A \rightarrow B$ is an equivalence and $T \subset A$, there is an object $S$ and an equivalence $\theta^{\prime}$ such that

$$
\theta[A \supset T]=[B \supset S] \theta^{\prime} .
$$

(v) If two idemmaps have the same range and the same domain, they are equal.

(vi) For each object $A$, the class of all $Q \leqq A$ is a set.

Note that axiom (v) includes the statement (11.3).

Every bicategory determines a bicategory with objects; conversely, the mappings of a bicategory with objects form a bicategory, if the injections are the mappings $[B \supset S]$ and the projection the mappings $[Q \leqq A]$.

In the canonical decomposition (11.4) call $S$ the image of $\alpha, Q$ the coimage of $\alpha$; in symbols,

$$
\operatorname{Im}(\alpha)=S \subset R(\alpha), \quad \operatorname{Coim}(\alpha)=Q \leqq D(\alpha) .
$$

By (vi), each object $A$ determines the $\operatorname{set} \delta(A)$ of all subobjects $T$, with $T \subset A$, and the set $2(A)$ of all quotients $R \leqq A$. Given $\alpha: A \rightarrow B$, each $T \subset A$ has an "image" $\alpha_{s} T \subset B$, and dually, as defined by

$$
\alpha_{s} T=\operatorname{Im}(\alpha[A \supset T]) \quad \alpha_{q} Q=\operatorname{Coim}([Q \leqq B] \alpha) .
$$

Then $\alpha_{s}$ is an (inclusion) order preserving transformation of the set $\mathcal{S}(A)$ into the set $\mathcal{S}(B)$, and, if $\alpha \beta$ is defined, $(\alpha \beta)_{s}=\alpha_{s} \beta_{s}$. One readily proves the following theorem.

THEOREM 11.6. If $\alpha$ is an identity map, $\alpha_{8}$ is the identity. If $\theta$ is an equivalence $\theta: A \rightarrow B, \theta_{s}$ is a one-to-one transformation of $S(A)$ onto $S(B)$, with inverse $\left(\theta^{-1}\right)_{s}$. If $\kappa$ is an injection $\kappa: S \rightarrow B, \kappa_{s}$ is the identity transformation of $\mathcal{S}(S)$ into $\mathcal{S}(B)$. If $\lambda: A \rightarrow B$ is a submapping, $\lambda_{s}$ 
is a one-to-one transformation of $\mathcal{S}(A)$ onto the subset $\mathcal{S}\left(\lambda_{s} A\right)$ of $\mathcal{S}(B)$.

A corresponding result for projections or supermappings does not hold in general. Note that the dual of Theorem 11.6 will assert, for example, that if $\rho: A \rightarrow B$ is a supermapping, then $\rho_{q}$ is a one-to-one transformation $2(B)$ onto a subset $2\left(\rho_{q} B\right)$ of $2(A)$-the logical phrases (one-to-one onto, and so on) are not changed by dualization.

12. Equality and examples of bicategories. We have already observed in $\$ 2$ that groups and homomorphisms, with the natural interpretation of an injection as the identity mapping applied to a subgroup and of a projection as a canonical homomorphism of $G$ on $G / N$, do not satisfy the axioms for a bicategory, because BC-2', on products of projections, fails. We avoid this difficulty by abandoning the current coset fashion for the classical congruence idea, and regarding $G / N$ as a group whose elements are simply the elements of $G$, with a new equality: $-g_{1} \equiv g_{2}(\bmod N)$ if and only if $g_{1} g_{2}^{-1} \in N$. A quotient group of a quotient group is then a quotient group.

More explicitly, we regard a group $G$ as the mathematical system $\left[M_{G},=_{G}, \times_{G}\right]$ consisting of a set $M_{G}$, a relation $=_{G}$, reflexive, symmetric, and transitive for the elements of $M_{G}$, and a binary operation $\times_{G}$ defined for all pairs of elements of $M_{G}$. To all the usual axioms and definitions we then append the appropriate equality axioms; for the group axioms they are

$$
\begin{aligned}
& a={ }_{G} b \text { implies } a \in M_{G}, \quad b \in M_{G}, \\
& a={ }_{G} b \text { and } g={ }_{G} h \text { imply } a \times \times_{G} g={ }_{G} b \times{ }_{G} h ;
\end{aligned}
$$

for a homomorphism $\alpha: G \rightarrow H$ they are

$$
\begin{aligned}
& a={ }_{G} b \text { implies } \alpha a={ }_{H} \alpha b, \\
& \alpha\left(a \times_{G} b\right)={ }_{H}(\alpha a) \times_{H}(\alpha b), \quad \text { for } a, b \in M_{G} ;
\end{aligned}
$$

for a subgroup $S \subset G$ they are

$$
\begin{array}{lr}
a={ }_{S} b \text { if and only if } a={ }_{G} b, & \text { for all } a \in M_{S}, \\
a \times{ }_{S} b={ }_{G} a \times{ }_{G} b, & \text { for all } a, b \in M_{S} .
\end{array}
$$

Finally, a quotient group $Q$ of $G$ is a group such that

$$
\begin{array}{lr}
h \in M_{Q} \text { implies } h \in M_{G}, & \\
a={ }_{G} b \text { implies } a={ }_{Q} b, & \text { for } a, b \in M_{G}, \\
a \times{ }_{G} b={ }_{Q} a \times{ }_{Q} b & \text { whenever } a, b \in M_{Q} .
\end{array}
$$

It follows that $M_{G}$ and $M_{Q}$ have the same elements. 
With similar definitions for the equality of groups and homomorphisms, and with an injection (projection) defined as a homomorphism $\alpha$ of a subgroup $S$ into $G$ (of $G$ upon a quotient group) determined by the identity function $j$, it readily follows that the class of all groups and homomorphisms constitutes a bicategory with objects. Similarly, we have the bicategory of all abelian groups, of all finite groups, of all rings, and so on.

The bicategory $\mathcal{S}$ of nonvoid sets will thus be interpreted as the bicategory of sets where each set $S$ carries with it an equivalence relation $=s$, and with the appropriate transformations as mappings. This bicategory has several special properties. The axiom of choice is equivalent to the theorem that for every mapping $\xi$ of $\mathcal{S}$ there is a mapping $\xi^{*}$ of $\delta$ with $\xi \xi^{*} \xi=\xi$. The left cancellable mappings are identical with the left invertible mappings and hence with the submappings (Lemma 11.4). The dual statement is also true. However, the dual of a true statement about the bicategory of sets need not always be true. For example, there exists a subclass $S^{*}$ of mappings which contains exactly one mapping with any given domain and any given range, and such that any right multiple of a mapping of $S^{*}$ is also in $S^{*}$. The dual assertion is false.

The bicategory of topological spaces has as objects all topological spaces and as mappings all continuous transformations of one such space into another. The definitions are again the standard ones, plus equality axioms; in particular $Q$ is a quotient space of $X$ if the set $M_{Q}$ is a quotient set of $M_{X}$, and if the identity transformation of $M_{X}$ onto $M_{Q}$ induces a continuous transformation of $X$ onto $Q$. Thus, if $X$ is decomposed into disjoint subsets, the usual decomposition space $Q$ may be interpreted as a quotient space of $X$-but these are not the only quotient spaces of $X$, since the same set $M_{Q}$ and the same equality may form a quotient space with fewer open sets. The axioms for a bicategory may be verified, the essential feature being the unique factorization axiom $\mathrm{BC}-3$, for a continuous transformation $\xi: X \rightarrow Y$. If we define the image $\xi(X) \subset Y$ with the usual relative topology, the set quotient of the transformation $\xi$ is then a set $Q \leqq X$ in one-to-one correspondence with the image space $\xi(X)$; using this correspondence we impose a topology on $Q$ which makes $Q$ a quotient space of $X$. This gives the factorization of $\xi$, the uniqueness following readily. The essential feature of this argument is the fact that every continuous image $\xi(X)$ of a topological space in another such is itself a topological space. We may thus speak of the bicategories of all $T_{0}, T_{1}, T_{2}$, or all compact Hausdorff spaces.

13. Universal algebra. These examples indicate that most types of 
algebraic, topological, or other mathematical systems, together with the appropriate type of transformations, yield bicategories. The bicategory language appears to be the appropriate vehicle for many of the theorems of universal algebra (cf. $[3 ; 4 ; 6 ; 15 ; 19 ; 20]$ ) -often giving simpler formulations, because the axiomatic formulation avoids the inevitably cumbersome explicit description of the general form of any algebraic or mathematical system. This is especially the case when universal algebra is extended to include those algebraic systems, which occur so frequently, in which several groups, homomorphisms, functions, and so forth, together constitute a single algebraic system. Using the notions of covariance and contravariance [8] one can in fact give a general definition of mathematical systems and prove, under general hypotheses, that the class of all systems of a given type is the class of objects of a bicategory.

The crucial point of this development is the definition of the "homomorphisms" appropriate to the type of system at hand. For example, a topological space may be regarded as a set $X$ together with a suitable, selected subset $V_{X}$ (open sets) of $2^{X}$. A homomorphism (continuous transformation) $\xi: X \rightarrow Y$ must then carry $\mho_{Y}$ into $V_{X}$; in other words $2^{X}$ must be regarded as a contravariant functor of $X$ (and not, as in some other cases, as a covariant functor). The general definition can then be extended to include algebraic systems defined by functors contravariant in one argument and covariant in another -a good illustrative example being the algebraic homotopy types considered in [17].

Leaving this development aside, we shall next show merely that the axioms for a bicategory can be extended so as to include also all the phenomena of universal algebra treated by lattice-theoretic means (the new axioms being valid in all standard examples).

14. Lattice ordered categories. A lattice ordered category (LC) is a bicategory $\mathcal{C}$ satisfying two ${ }^{8}$ additional axioms (and their duals).

LC-1. For each object $A$, every nonvoid subset of $\mathcal{S}(A)$ has a least upper bound (l.u.b.) in the partially ordered set $\mathcal{S}(A)$.

Here $S(A)$ denotes the set of all subobjects $S, T, \cdots$ of $A$ with the partial order $S \subset T$. The l.u.b. of a collection $\left\{S_{i}\right\}$ of subobjects will be denoted by $\sum_{A} S_{i}$, that of two subobjects by $S_{1} \cup_{A} S_{2}$.

LC-2. If $\alpha: A \rightarrow B$ and $\left\{S_{i}\right\}$ is a nonvoid subset of $S(A)$,

$$
\alpha_{s}\left(\sum_{A} S_{i}\right) \subset \sum_{B} \alpha_{8} S_{i} .
$$

${ }^{8}$ It might be desirable to add an axiom LC-3 (and its dual) requiring that $\alpha_{1}, \alpha_{2}: A \rightarrow B, A=\sum_{A} S_{i}$, and $\alpha_{1}\left[A \supset S_{i}\right]=\alpha_{2}\left[A \supset S_{i}\right]$ for all $i$ imply that $\alpha_{1}=\alpha_{2}$. 
Since $\alpha_{s}$ preserves order, we deduce that equality actually holds in (14.1). A nonvoid set of objects $S_{i}$ with upper and lower bounds $C \subset S_{i} \subset A$ has, by the usual proof, a g.1.b. $\prod S_{i}$ which is independent of the choice of $C$ and $A$. If $\alpha: A \rightarrow B$, then $\alpha_{s}\left(\prod S_{i}\right) \subset \prod \alpha_{s} S_{i}$, with equality when $\alpha$ is a submapping (the latter by Theorem 11.6). The set of all $S$ with $C \subset S \subset A$ is a complete lattice [5]. The 1.u.b. $\sum_{A} S_{i}$ may depend on the choice of the "universe" $A$, but

$$
\sum_{A} S_{i}=\sum_{B} S_{i}, \quad \text { if } S_{i} \subset A \subset B, \text { all } i .
$$

If we use only LC-1, the inverse image of any subobject $T \subset B$ under a mapping $\alpha: A \rightarrow B$ is defined as

$$
\alpha_{s}^{*} T=\sum_{A} S, \quad \text { over all } S \subset A \text { with } \alpha_{s} S \subset T .
$$

In this notation LC-2 is equivalent to the requirement that

$$
\alpha_{s} \alpha_{s}^{*} T \subset T, \quad \text { for all } \alpha: A \rightarrow B, T \subset B .
$$

Also, $S=\alpha_{s}^{*} T$ is characterized as a subobject of $A$ by the properties (i) $\alpha_{8} S \subset T$; (ii) $\alpha_{s} S^{\prime} \subset T$ for $S^{\prime} \subset A$ implies $S^{\prime} \subset S$. Furthermore, $\alpha_{s}^{*}$ is an order preserving transformation of $\mathcal{S}(B)$ into $\mathcal{S}(A)$, with $(\alpha \beta)_{s}^{*}$ $=\beta_{s}{ }^{*} \alpha_{s}{ }^{*}$ whenever $\alpha \beta$ is defined. One has also the following theorem.

Theorem 14.1. If $\alpha: A \rightarrow B$ is an identity, so is $\alpha_{s}^{*}$; if $\alpha$ is an equivalence, $\alpha_{s}^{*}$ is a one-to-one transformation of $\mathcal{S}(B)$ onto $\mathcal{S}(A)$, with $\alpha_{s}$ as inverse, while if $\alpha$ is a submapping, $\alpha_{s}^{*}$ maps $\mathcal{S}(B)$ onto $\mathcal{S}(A)$. If $\kappa=[A \supset S]$ is an injection, and $T \subset A$, then $\kappa_{s}^{*} T=T \cap S$.

The dual statements hold; in particular any two quotients $Q_{1} \leqq A$ and $Q_{2} \leqq A$ have an l.u.b. $Q_{1} \vee Q_{2}$ and a g.l.b. $Q_{1} \wedge Q_{2}$, the latter whenever they have some lower bound. For $\alpha: A \rightarrow B$ the inverse coimage $\alpha_{q}^{*}$ yields a transformation of $2(A)$ into $2(B)$.

The notion of a lattice ordered category is a simultaneous generalization of the notions of group and lattice; specifically, every group is a lattice ordered category, in which every mapping is an equivalence and there is only one identity, and every lattice is a lattice ordered category in which every mapping is an injection (or dually).

15. Zeros and zero mappings. A category $\mathcal{C}$ has a zero if it satisfies the following axiom.

$Z$. There is an object $Z$ such that for every object $A$ of $\mathcal{C}$ there exists exactly one mapping $\zeta: A \rightarrow Z$ and exactly one mapping $\eta: Z \rightarrow A$. We call $Z$ a zero object. 
In the category of groups (abelian groups, rings) any group with only the identity element (ring with only the zero) is a zero in this sense. The category of topological space does not satisfy axiom $Z$; however that of topological spaces with a distinguished base point (as used in homotopy theory) does. The investigations [15] of Jónsson and Tarski have already indicated that the presence (or absence) of a zero is of critical importance in many investigations of universal algebra. ${ }^{9}$

Given axiom $Z$, it follows at once that any two zero objects are equivalent. A mapping $\alpha$ is said to be a zero mapping if it can be factored as $\alpha=\eta \zeta$, where $R(\zeta)=D(\eta)=Z$. This definition is independent of the choice of the zero $Z$. Given any two objects $A$ and $B$ in the category, there is exactly one zero mapping $\alpha: A \rightarrow B$; we denote this mapping as $0_{B A}$, so that $0_{C B} 0_{B A}=0_{C A}$. In the category of all groups $0_{B A}$ is the homomorphism mapping all elements of $A$ into the identity element of $B$. If $\alpha=0$ (that is, if $\alpha$ is a zero mapping), then $\alpha \beta=0$ and $\gamma \alpha=0$ whenever these products are defined.

Under the axioms BCZ (bicategory with a zero) we define, for each object $A$,

$$
1_{A}=\operatorname{Im}\left(0_{A Z}\right), \quad 1_{A}^{\prime}=\operatorname{Coim}\left(0_{Z A}\right) .
$$

For groups, $1_{A}$ is the identity subgroup of $A$, and $1_{A}$ the quotient group $A / A$. In general (BCZ) $1_{A} \subset A$; for $S \subset A, 1_{A} \subset S \subset A$; and $1_{A}$ is a zero object. The mapping $0_{Z_{A}}$ is a supermap, while $0_{A Z}$ is a submap, and any zero mapping $0_{B A}$ has

$$
0_{B A}=\left[B \supset 1_{B}\right] 0_{1_{B} 1_{A}^{\prime}}\left[1_{A}^{\prime} \leqq A\right]
$$

as its canonical decomposition.

LemmA 15.1 (BCZ). If $\lambda$ is a submap and $\rho$ a supermap, then

$$
\lambda \beta=0 \text { implies } \beta=0 ; \quad \alpha \rho=0 \text { implies } \alpha=0 .
$$

Proof. Since $\lambda$ is a submap, $\lambda=\kappa \theta$ with $\kappa$ an injection, $\theta$ an equivalence. Let $\theta \beta$ have the canonical decomposition $\theta \beta=\kappa_{1} \theta_{1} \pi_{1}$. Then $\lambda \beta=\kappa \theta \beta$ has the canonical decomposition $\left(\kappa \kappa_{1}\right) \theta_{1} \pi_{1}$. This must agree with a canonical decomposition of the form (15.2), whence $\theta_{1}=0$ and $\beta=\theta^{-1}(\theta \beta)=\theta^{-1} \kappa_{1} \theta_{1} \pi_{1}=0$.

Finally, under the axioms LCZ (lattice ordered category with $Z$ ) we observe that each $\mathcal{S}(A)$ and each $\mathscr{2}(A)$ is a complete lattice (with unit $1_{A}$ resp. $\left.1_{A}^{\prime}\right)$ and that

${ }^{9}$ The Jonsson-Tarski zeros give a zero in the sense of this axiom, but the author has been unable to prove their direct decomposition theorems in categorical form. 


$$
\alpha: A \rightarrow B \text { implies } \alpha_{s} 1_{A}=1_{B}, \alpha_{q} 1_{B}^{\prime}=1_{A}^{\prime} .
$$

The axioms LCZ hold in the bicategories of all groups, of all rings, of all vector spaces over a fixed division ring, and so on.

16. Normality and kernels. A categorical definition of normal subobject can be formulated conveniently under the axioms LCZ. For subobjects $S \subset A$ and quotient objects $Q \leqq A$ we define an orthogonality relation

$$
S \perp_{A} Q \quad \text { if and only if }[Q \leqq A][A \supset S]=0 \text {; }
$$

using the axiom LC-1 we then give dual definitions:

$$
\begin{gathered}
A / S=\text { l.u.b. in } 2(A) \text { of all } Q \leqq A \text { with } S \perp_{A} Q, \\
A \div Q=1 . \text { u.b. in } S(A) \text { of all } S \subset A \text { with } S \perp_{A} Q .
\end{gathered}
$$

For groups, $A / S$ will then be the usual factor group $A / N$, where $N \supset S$ is the least normal subgroup of $A$ containing $S$. In general, one then proves, using LC-2 in (16.5), that

$$
\begin{array}{ll}
A / 1_{A}=A, & A / A=1_{A}^{\prime}, \\
S \perp_{A}(A / S), & (A \div Q) \perp_{A} Q, \\
S_{1} \subset S_{2} \quad \text { implies } & A / S_{2} \leqq A / S_{1}, \\
S \subset A \div(A / S), & A / S=A /(A \div(A / S)) ;
\end{array}
$$

in other words $S \rightarrow A / S, Q \rightarrow A \div Q$ provides a Galois connection [5, p. 56] between $S(A)$ and $2(A)$.

Finally, we define

(16.8) $S$ is normal in $A$ if and only if $S=A \div(A / S)$,

and dually. Alternative definitions are given by the following theorem.

THeORem 16.1. A subobject $S \subset A$ is normal in $A$ if and only if any one of the following conditions hold.

(i) There exists $Q \leqq A$ such that $S=A \div Q$;

(ii) There exists a $Q$ conormal in $A$ such that $S=A \div Q$;

(iii) If, for all $Q, S \perp_{A} Q$ implies $S^{\prime} \perp_{A} Q$, then $S^{\prime} \subset S$;

(iv) If, for all $\alpha: A \rightarrow B, \alpha[A \supset S]=0$ implies $\alpha\left[A \supset S^{\prime}\right]=0$, then $S^{\prime} \subset S$

(v) There exists a $Q \leqq A$ such that $S \perp_{A} Q$ and $S^{\prime} \subset S$ whenever $S^{\prime} \perp_{A} Q$.

The proof of (iv) makes essential use of Lemma 15.1. Also, it may readily be proved that $1_{A}$ and $A$ are normal in $A$, that $N \subset T \subset A$ with $N$ normal in $A$ implies $N$ normal in $T$, and that the intersection 
$\pi N_{i}$ of objects normal in $A$ is normal in $A$. (Apparently the similar assertion for union, though true for groups, is not a consequence of the axiom LCZ.)

For any mapping $\alpha: A \rightarrow B$ the kernel $K(\alpha)$ may be defined as

$$
K(\alpha)=A \div(\operatorname{Coim} \alpha)=\alpha_{s}^{*} 1_{B} .
$$

Then a subobject $S$ is normal in $A$ if and only if it is the kernel of some $\alpha$; when so, it is in fact the kernel of $[A / S \leqq A]$. Furthermore $K(\alpha \beta) \supset K(\beta)$, and, for a canonical decomposition, $K(\kappa \theta \pi)=K(\pi)$.

A further development giving the first and second isomorphism theorems, and so on, can be made by introducing additional carefully chosen dual axioms. This will be done below only in the more symmetrical abelian case. It is also possible to give a definition of normality in lattice ordered categories without a zero, by using the criterion (iv) of Theorem 16.1, with the zero object suitably replaced by objects acting like spaces or sets with only one element. This definition applies in any category, agrees with the above definition when a zero is present, and in the category of $T_{1}$ spaces has the amusing property that the normal subobjects of a topological space are exactly the closed subsets of that space! (Because of the failure of separation axioms in a decomposition space, this is not true in the category of $T_{2}$ spaces.) However, in the category of all compact Hausdorff spaces (with mappings all continuous transformations) every subspace (being closed) is normal, but there are nonconormal quotient spaces. This reverses the group-theoretic phenomenon that every quotient group is conormal.

\section{Abelian categories}

17. The group of integers. Our objective in this chapter is that of providing a self-dual set of axioms for abelian groups and their homomorphisms sufficient to prove all categorical theorems which refer to a finite number of such groups - and hence adequate to explain the apparent perfect duality present for such theorems on abelian groups. We also obtain a representation theorem for certain abstract categories, using the following purely categorical characterization of the additive group of integers.

Definition. An object $J$ in a category $\mathcal{C}$ is integral in $\mathcal{C}$ if

(i) For two distinct mappings $\alpha_{1}, \alpha_{2}: G \rightarrow H$ of $\mathcal{C}$ with the same domain $G$ and the same range $H$ there exists a mapping $\beta: J \rightarrow G$ such that $\alpha_{1} \beta \neq \alpha_{2} \beta$.

(ii) If $J^{\prime}$ is another object of $\mathcal{C}$ with the property (i), there exists in $\mathcal{C}$ a mapping $\sigma: J^{\prime} \rightarrow J$ with a right inverse in $\mathcal{C}$. 
(iii) If $\alpha_{1} \alpha_{2}=I_{J}$ for two mappings $\alpha_{1}, \alpha_{2}: J \rightarrow J$, then $\alpha_{1}$ and $\alpha_{2}$ are equivalences.

One then readily proves the following theorems.

THEOREM 17.1. The additive group of integers is integral (satisfies (i), (ii), and (iii)) in the category of all abelian groups.

THEOREM 17.2. In any category, any two objects with the properties (i), (ii), and (iii) are equivalent.

Proof. Given $J, J^{\prime}$ both integral, there exist by (ii) mappings $\sigma: J^{\prime} \rightarrow J$ and $\tau: J \rightarrow J^{\prime}$, each with right inverse. Then $\tau \sigma: J^{\prime} \rightarrow J^{\prime}$, and $\sigma \tau: J \rightarrow J$ have right inverses, hence by (iii) are equivalences. Therefore $(\tau \sigma)^{-1} \tau \sigma=I_{J^{\prime}}$, and $\sigma$ has a left inverse; therefore $\sigma$ is an equivalence $\sigma: J^{\prime} \rightarrow J$, as asserted.

A somewhat more difficult proof gives the following theorem.

THEOREM 17.3. The additive group L of rational numbers modulo 1 is cointegral (satisfies the duals of (i), (ii), and (iii)) in the category of all abelian groups.

Hence the group $L$ can also be characterized up to isomorphism. We note by the way that the space consisting of one point only is integral in the category of all topological spaces, but we do not know of (and doubt the existence of) a "cointegral" object in this category. However, in the category of all vector spaces over a fixed division ring $D$, the vector space $D$ is both integral and cointegral.

18. Abelian categories. For abelian groups and for vector spaces the cartesian product (of a finite number of factors) can be regarded simultaneously as a free and a direct product, in the sense of $\$ 3$ above. We thus introduce corresponding axioms on a category.

Definition. In a category with zero, a (simultaneous) free-anddirect product of objects $A$ and $B$ is a diagram

$$
A \leftrightarrows A \times B \rightleftarrows B
$$

consisting of an object $A \times B$ and four mappings $\Gamma^{1}, \Gamma^{2}, \Lambda^{1}, \Lambda^{2}$,

$$
\begin{array}{ll}
\Gamma_{A \times B}^{1}: A \times B \rightarrow A, & \Gamma_{A \times B}^{2}: A \times B \rightarrow B, \\
\Lambda_{A \times B}^{1}: A \rightarrow A \times B, & \Lambda_{A \times B}^{2}: B \rightarrow A \times B,
\end{array}
$$

with the following three properties (omitting the subscript $A \times B$ to simplify notation):

(i) $\Gamma^{1} \Lambda^{1}=I_{A}, \Gamma^{1} \Lambda^{2}=0_{A B}, \Gamma^{2} \Lambda^{1}=0_{B A}, \Gamma^{2} \Lambda^{2}=I_{B}$;

(ii) For any pair of mappings $\alpha_{1}: C \rightarrow A, \alpha_{2}: C \rightarrow B$, there exists a 
unique $\gamma: C \rightarrow A \times B$ with $\Gamma^{1} \gamma=\alpha_{1}, \Gamma^{2} \gamma=\alpha_{2}$;

(iii) For any pair of mappings $\beta_{1}: A \rightarrow D, \beta_{2}: B \rightarrow D$, there exists a unique $\delta: A \times B \rightarrow D$ with $\delta \Lambda^{1}=\beta_{1}, \delta \Lambda^{2}=\beta_{2}$.

Here property (ii) asserts that we have a direct product and (iii) that we have a free product, both in the sense of $\$ 3$.

Definition. An abelian category (AC) is a category $\mathcal{C}$ satisfying the axiom $Z$ (existence of zero) and the axioms

AC-1. There exists an integral and a cointegral object in $\mathcal{C}$.

AC-2. There exists in $\mathcal{C}$ a free-and-direct product for any two objects of $\mathcal{C}$.

Theorem 18.1. Any two free-and-direct products of two objects $A$ and $B$ have isomorphic diagrams (18.1).

If $A * B$ is the second product, the isomorphism of the diagrams means that there is an equivalence mapping $\theta: A \times B \rightarrow A * B$ with

$$
\theta \Lambda_{A \times B}^{i}=\stackrel{i}{i}, \quad \Gamma_{A * B}^{i}, \quad \Gamma_{A * B}^{i} \theta=\Gamma_{A \times B}^{i}, \quad i=1,2 .
$$

The proof is straightforward. This result and a similar theorem for triple products will prove the following theorem.

THEOREM 18.2. The operation of forming free-and-direct products is commutative and associative (in the sense of diagram isomorphism).

This operation also has the zero object $Z$ as identity, as follows:

Theorem 18.3. The diagram

$$
G \leftrightarrows G \rightleftarrows Z
$$

with identity mappings on the left, zero mappings on the right, represents $G$ as the free-and-direct product $G \times Z$.

Two homomorphisms $\alpha_{1}, \alpha_{2}$ for groups have a cartesian product defined by

$$
\left(\alpha_{1} \times \alpha_{2}\right)(a, b)=\left(\alpha_{1} a, \alpha_{2} b\right) .
$$

This product may be introduced on the basis of our axioms.

Theorem 18.4. Given mappings $\alpha_{1}: A \rightarrow A^{\prime}, \alpha_{2}: B \rightarrow B^{\prime}$, there exists one and only one mapping $\alpha_{1} \times \alpha_{2}: A \times B \rightarrow A^{\prime} \times B^{\prime}$ with

$$
\Gamma_{A^{\prime} \times B^{\prime}}^{i}\left(\alpha_{1} \times \alpha_{2}\right)=\alpha_{i} \Gamma_{A \times B}^{i},\left(\alpha_{1} \times \alpha_{2}\right) \Lambda_{A \times B}^{i}=\Lambda_{A^{\prime} \times B^{\prime}}^{i} \alpha_{i}, i=1,2 .
$$

The mapping $\alpha_{1} \times \alpha_{2}$ is determined by either one of these two equations. 
The proof (here and elsewhere) may be visualized most readily by drawing the appropriate diagrams. By (ii), there exists a unique mapping $\gamma=\alpha_{1} \times \alpha_{2}$ with the first property of (18.4). Then by (i)

$$
\begin{aligned}
\Gamma^{i}\left(\alpha_{1} \times \alpha_{2}\right) \Lambda^{j}=\alpha_{i} \Gamma^{i} \Lambda^{j} & =\alpha_{i} & & (\text { for } i=j) \\
& =0 & & (\text { for } i \neq j) .
\end{aligned}
$$

But on the other hand, by (i),

$$
\begin{aligned}
\Gamma^{\prime}{ }^{\prime} \Lambda^{\prime j} \alpha_{j} & =\alpha_{i} & & (\text { for } i=j) \\
& =0 & & (\text { for } i \neq j) .
\end{aligned}
$$

Hence, by the uniqueness assertion of (ii) for $A^{\prime} \times B^{\prime}$, the second equation of (18.4) holds.

One shows also that $0 \times 0=0$ and $I_{A} \times I_{B}=I_{A \times B}$.

For the cartesian square $A \times A$ of an abelian group there is a diagonal homomorphism

$$
\nabla_{A}: A \rightarrow A \times A \quad \text { with } \nabla_{A}(a)=(a, a)
$$

and a codiagonal homomorphism

$$
\Delta_{A}: A \times A \rightarrow A \quad \text { with } \Delta_{A}(a, b)=a+b .
$$

Under our axioms, these mappings can be characterized dually as the unique mappings such that

$$
\begin{array}{lll}
\nabla_{A}: A \rightarrow A \times A, & \Gamma_{A \times A}^{i} \nabla_{A}=I_{A}, & i=1,2 ; \\
\Delta_{A}: A \times A \rightarrow A, & \Delta_{A} \Lambda_{A \times A}^{i}=I_{A}, & i=1,2 .
\end{array}
$$

The mappings $\Gamma^{i}, \Lambda^{i}, \gamma$, and $\delta$ appearing in the definition of the freeand-direct product may then be expressed as

$$
\begin{aligned}
\Gamma^{1} & =\Delta_{A}\left(I_{A} \times 0_{A B}\right), & \Gamma^{2} & =\Delta_{B}\left(0_{B A} \times I_{B}\right), \\
\Lambda^{1} & =\left(I_{A} \times 0_{B A}\right) \nabla_{A}, & \Lambda^{2} & =\left(0_{A B} \times I_{B}\right) \nabla_{B}, \\
\gamma & =\left(\alpha_{1} \times \alpha_{2}\right) \nabla_{C}, & \delta & =\Delta_{D}\left(\beta_{1} \times \beta_{2}\right) .
\end{aligned}
$$

THEOREM 18.5. The axiom AC-2 asserting the existence of free-anddirect products may be replaced by the assumption that there exists to each pair of objects an object $A \times B$, to each pair of mappings $\alpha_{1}: A \rightarrow A^{\prime}$, $\alpha_{2}: B \rightarrow B^{\prime}$ a mapping $\alpha_{1} \times \alpha_{2}: A \times B \rightarrow A^{\prime} \times B^{\prime}$, and to each object $A$ two mappings $\Delta_{A}: A \times A \rightarrow A, \nabla_{A}: A \rightarrow A \times A$ such that

(i) $\left(\alpha_{1} \times \alpha_{2}\right)\left(\beta_{1} \times \beta_{2}\right)=\left(\alpha_{1} \beta_{1}\right) \times\left(\alpha_{2} \beta_{2}\right)$ whenever $\alpha_{1} \beta_{1}$ and $\alpha_{2} \beta_{2}$ are defined;

(ii) If $\alpha: A \rightarrow B$, then

$$
\Delta_{B}\left(\alpha \times 00_{B A}\right) \nabla_{A}=\alpha=\Delta_{B}\left(0_{B A} \times \alpha\right) \nabla_{A} ;
$$


(iii) Every $\gamma: C \rightarrow A \times B$ has the form

$$
\gamma=(\alpha \times \beta) \nabla_{C} \quad \text { for some } \alpha: C \rightarrow A, \beta: C \rightarrow B ;
$$

(iii') Every $\delta: A \times B \rightarrow C$ has the form

$$
\delta=\Delta_{C}(\alpha \times \beta) \quad \text { for some } \alpha: A \rightarrow C, \beta: B \rightarrow C .
$$

Proof. Given AC-2, (i) is readily derived, while (ii) results from the equations

$$
\begin{aligned}
\Delta_{B}\left(\alpha \times 0_{B A}\right) \nabla_{A} & =\Delta_{B}\left(\alpha \times 0_{B Z}\right)\left(I_{A} \times 0_{Z A}\right) \nabla_{A} \\
& =\left\{\Delta_{B}\left(\alpha \times 0_{B Z}\right) \Lambda_{A \times Z}^{1}\right\}\left\{\Gamma_{A \times Z}^{1}\left(I_{A} \times 0_{Z A}\right) \nabla_{A}\right\} \\
& =\left\{\Delta_{B} \Lambda_{B}^{1} \times{ }_{B} \alpha\left\{I_{A} \Gamma_{A}^{1} \times{ }_{A} \nabla_{A}\right\}\right. \\
& =\left\{I_{B} \alpha\right\}\left\{I_{A} I_{A}\right\}=\alpha .
\end{aligned}
$$

'To prove (iii) we set $\alpha=\Gamma_{A \times B}^{1} \gamma, \beta=\Gamma_{A \times B}^{2} \gamma$ and apply the appropriate definitions; ( $\mathrm{iii}^{\prime}$ ) is the dual.

Conversely, given the conditions (i) to (iii'), we use the definitions (18.6), (18.6'), and (18.7) to construct the free-and-direct product diagram on $A \times B$ and to prove its properties.

The "existential" character of condition (iii) in this theorem, corresponding to the existential character of (ii) in (18.2), can be avoided, by using the readily proved identities

$$
\begin{aligned}
& (\alpha \times \alpha) \nabla_{A}=\nabla_{B} \alpha, \quad \Delta_{B}(\alpha \times \alpha)=\alpha \Delta_{A}, \quad \text { for } \alpha: A \rightarrow B, \\
& {\left[\Delta_{A}\left(I_{A} \times 0_{A B}\right)\right] \times\left[\Delta_{B}\left(0_{B A} \times I_{B}\right)\right] \nabla_{A} \times{ }_{B}=I_{A} \times_{B} .}
\end{aligned}
$$

THEOREM 18.6. Theorem 18.5 remains valid if conditions (iii) and (iii') are replaced by (18.8), (18.9), and the dual of (18.9).

19. Addition of mappings. Two homomorphisms $\alpha, \beta: A \rightarrow B$ for abelian groups $A$ and $B$ have a sum $\alpha+\beta: A \rightarrow B$ defined by $(\alpha+\beta)(a)$ $=\alpha(a)+\beta(a)$. For groups one readily verifies that this sum can be expressed in terms of diagonal mappings, and so on, as

$$
\alpha+\beta=\Delta_{B}(\alpha \times \beta) \nabla_{A} \text {. }
$$

We adopt the self-dual expression (19.1) as the definition of the sum of two coterminal mappings in any abelian category. By suitable use of the Uniqueness Theorem 18.1, one shows that the sum is independent of the choice of the free-and-direct products $B \times B$ and $A \times A$ entering its definition. For $0=0_{B A}$ the equation

$$
0+\beta=\beta=\beta+0
$$

follows from Theorem 18.3. The commutative and associative laws 


$$
\alpha+\beta=\beta+\alpha, \quad(\alpha+\beta)+\gamma=\alpha+(\beta+\gamma)
$$

similarly follow from commutative and associative properties of the product $A \times B$, using a lengthy but straightforward manipulation of diagrams. One may also establish the distributive laws

$$
(\alpha+\beta) \gamma=\alpha \gamma+\beta \gamma, \quad \gamma(\alpha+\beta)=\gamma \alpha+\gamma \beta,
$$

each valid whenever the left side is defined. A similar but lengthy proof shows that the mappings $\Gamma^{i}, \Lambda^{i}$ entering the definitions (18.2) of the product satisfy the equation

$$
\Lambda^{1} \Gamma^{1}+\Lambda^{2} \Gamma^{2}=I_{A \times B} .
$$

This gives an alternative characterization of the direct-and-free product as follows.

Theorem 19.1. A diagram (18.1) in an abelian category, with mappings satisfying (18.1), (18.2), condition (i) below (18.2), and (19.5) is isomorphic to the free-and-direct product diagram for $A$ and $B$.

In an abelian category which is also a bicategory we have $\Gamma^{i} \Lambda^{i}$ $=$ identity by (i) of (18.2), hence by Lemma 11.4, the mappings $\Gamma^{i}$ appearing in a free-and-direct product are supermaps, the mappings $\Lambda^{i}$ are submaps (exactly as in the usual group-theoretic case). Similarly, by (18.5), $\Delta_{A}$ is a supermap, and $\nabla_{A}$ a submap.

20. The representation theorem. We understand an abelian semigroup $G$ to be a set closed under a commutative and associative operation of addition and containing a zero for this operation (we do not assume a cancellation law). The class of all abelian semigroups and all homomorphisms of one such into another (with or without the precautions of $\$ 12$ as to equality) is then a category. Our representation theorem now is

Theorem 20.1. Any abelian category $C$ is isomorphic to a category of semigroups.

Proof. Let $J$ be the essentially unique integral object of $C$. To each object $A$ of $\mathcal{C}$ assign the semigroup $G_{A}$ consisting of all mappings $\xi: J \rightarrow A$ in $\mathcal{C}$, with addition defined as in $\S 19$. Then $G_{A}$ is a semigroup with $0_{A J}$ as zero. To each mapping $\alpha: A \rightarrow B$ construct the transformation $M_{\alpha}: G_{A} \rightarrow G_{B}$ defined by $M_{\alpha} \xi=\alpha \xi: J \rightarrow B$, for each $\xi$ in $G_{A}$. By the distributive law (19.4), $M_{\alpha}$ is a semigroup homomorphism. By the first property of an integral object, $\alpha_{1} \neq \alpha_{2}: A \rightarrow B$ implies $M_{\alpha_{1}} \neq M_{\alpha_{2}}$. By the associative law in a category, $M_{\alpha \beta}=M_{\alpha} M_{\beta}$ whenever $\alpha \beta$ is defined. Hence the correspondence $A \rightarrow G_{A}, \alpha \rightarrow M_{\alpha}$ provides the desired isomorphic representation of the category $\mathcal{C}$. 
This theorem shows that our axioms for an abelian category include all the "purely formal" properties of homomorphisms for abelian groups, except for properties dependent upon the existence of the inverse.

21. Abelian bicategories. By an abelian bicategory ( $A B C$ ) we understand a lattice ordered bicategory (\$14) which is also an abelian category $(\$ 18)$ and which is subject to the following additional axioms ${ }^{10} \mathrm{ABC}-1$ to $\mathrm{ABC}-5$.

ABC-1. For each object $A$ there exists a map $V_{A}: A \rightarrow A$ such that $V_{A}+I_{A}=0_{A A}$.

It follows readily that $V_{A}$ is unique, and that $V_{A} V_{A}=I_{A}$. Furthermore, if $\alpha: A \rightarrow B$, then $\alpha V_{A}+\alpha=\alpha\left(V_{A}+I_{A}\right)=0$ and $V_{B} \alpha+\alpha=0$, hence $V_{B} \alpha=\alpha V_{A}$. We may thus define the (additive) inverse of $\alpha$ as $(-\alpha)=\alpha V_{A}=V_{B} \alpha$; it has the properties

$$
\alpha+(-\alpha)=0, \quad(-\alpha) \beta=-(\alpha \beta)=\alpha(-\beta) .
$$

Moreover, it may be shown, using (18.8), that the kernel of $\Delta_{A}$ is the image of $\left(I_{A} \times V_{A}\right) \nabla_{A}: A \rightarrow A \times A$, as in the case of groups.

ABC-2. If $\kappa_{1}, \kappa_{2}$ are injections, so is $\kappa_{1} \times \kappa_{2}$, and dually.

This gives the canonical decomposition of any $\alpha_{1} \times \alpha_{2}$; it is not used further below.

ABC-3. Every subobject is normal (in the sense of $\$ 16$ ) and dually. ABC-4. If $A \supset S \supset T$, then $S / T \subset A / T$, and dually.

ABC-5. If $A \supset T, A / T \supset M$, then $M=S / T$ for some $S$ with $A \supset S$ $\supset T$, and dually.

$\mathrm{ABC}-3$ and the dual of ABC-5 fail in the category of all groups ${ }^{10}$ but hold for abelian groups. Using them, one can prove that any projection $\pi: A \rightarrow Q$ with kernel $K(\pi)=K$ has $Q=A / K$, and hence that the canonical decomposition of any mapping $\alpha: A \rightarrow B$ has the customary form

$$
\alpha=[B \supset \operatorname{Im} \alpha] \theta[A / K(\alpha) \leqq A] .
$$

Furthermore, if $T \subset B$ and $S \subset A$;

$$
\alpha_{q}(B / T)=A / \alpha_{s}^{*} T, \quad \alpha_{q}^{*}(A / S)=B / \alpha_{s} S
$$

and dually. If $A \supset S \supset T$ with $A \neq S$, then $A / T \neq S / T$. If $\kappa=[A \supset S]$ is an injection, the induced transformations $\kappa_{s}$, and so forth, are given

\footnotetext{
${ }^{10}$ Axiom $\mathrm{ABC}-3$ and its dual are valid in the category of all groups under the hypothesis that $\pi=[A / T \leqq A]$ is a "conormal" mapping-that is, that $\pi_{q}$ carries conormal quotients of $A / T$ into conormal quotients of $A$. By this and analogous devices the proofs of the first and second isomorphism theorems for all groups can be based on "categorical" axioms.
} 
for any $T \subset S$ and any $U \subset A$ by the formulas

$$
\begin{aligned}
{[A \supset S]_{s} T } & =T, & {[A \supset S]_{s}^{*} U } & =U \cap S, \\
{[A \supset S]_{q}(A / U) } & =S / U \cap S, & {[A \supset S]_{q}^{*}(S / T) } & =A / T .
\end{aligned}
$$

If $\left\{S_{i}\right\}$ is a nonvoid collection of subobjects of $A$, then

$$
\sum_{A}\left(A / S_{i}\right)=A / \Pi S_{i}, \quad \Pi\left(A / S_{i}\right)=A / \sum_{A} S_{i} .
$$

The second isomorphism theorem holds in the form

$$
A \supset S \supset T \text { implies } A / S=(A / T) /(S / T) ;
$$

the proof using the fact that

$$
[A / T \supset S / T][S / T \leqq S]=[A / T \leqq A][A \supset S],
$$

by $\mathrm{ABC}-4$ and $\mathrm{BC}-5$. (This is a typical indication of the force of $\mathrm{BC}-5$.)

The first isomorphism theorem also holds in the form that

$$
A \supset S, A \supset N \text { imply }(S \cup N) / N \cong S / S \cap N,
$$

where the isomorphism in question is the equivalence factor of the mapping $[A / N \leqq A][A \supset S]$. If $\pi=[A / M \leqq A]$ is a projection, the induced transformations $\pi_{\varepsilon}$, and so on, are given by formulas analogous to (21.3), for $M \subset T \subset A, L \subset A$, as

$$
\begin{aligned}
& {[A / M \leqq A]_{q}(A / T)=A / T, \quad[A / M \leqq A]_{q}^{*}(A / L)=A / M \cup L,} \\
& {[A / M \leqq A]_{s} L=(M \cup L) / M,[A / M \leqq A]_{s}^{*}(T / M)=T}
\end{aligned}
$$

In none of these results is ABC-5 used, except in the last case to justify the representation of any subobject of $A / M$ in the form $T / M$.

In axiom ABC-5, one may show that the $S$ whose existence is asserted actually has the form $\pi_{s}^{*} M$, where $\pi=[A / T \leqq A]$, and thence that $\pi_{s}^{*} \pi_{s} S=S$.

In an ABC-category the class of submappings is entirely determined by the category structure (without intervention of the injections) according to the following readily proved result.

Theorem 21.1 (ABC). A mapping $\alpha$ is a submapping if and only if it is left cancellable.

Furthermore, if $\rho$ is a supermapping $\rho: A \rightarrow B$, the transformation $\rho_{s}^{*}$ is a right inverse of $\rho_{s}: \mathcal{S}(A) \rightarrow \mathcal{S}(B)$, and if $\lambda$ is a submapping $\lambda: A \rightarrow B, \lambda_{s}^{*}$ is a left inverse of the transformation $\lambda_{s}: \mathcal{S}(A) \rightarrow \mathcal{S}(B)$. Note that these statements are not dual to each other-though the 
dual statements about $\lambda_{q}, \lambda_{q}^{*}, \rho_{q}, \rho_{q}^{*}$ are, of course, also demonstrable. Another pair of such pseudo-dual statements is indicated by the following theorem.

TheOREM 21.2 (ABC). A mapping $\alpha$ is a supermapping if and only if either $\alpha_{s}$ is a transformation of $S(A)$ onto $S(B)$ or $\alpha_{s}^{*}$ is a one-to-one transformation of $\mathcal{S}(B)$ into $\mathcal{S}(A)$.

One may also establish the "extension equivalence" theorem. As in (1.3) an extension of $G$ by $Q$ is a diagram

$$
G \stackrel{\lambda}{\rightarrow} E \stackrel{\rho}{\rightarrow} Q
$$

such that $\lambda$ is a submapping, $\rho$ a supermapping, and image $(\lambda)$ $=$ kernel $(\rho)$. If

$$
G \stackrel{\lambda^{\prime}}{\rightarrow} E^{\prime} \stackrel{\rho^{\prime}}{\rightarrow} Q
$$

is a second such extension (with the same $G$ and $Q$ ), a "homomorphism" of (21.8) into (21.9) is given by any mapping $\gamma: E \rightarrow E^{\prime}$ which satisfies the usual commutation relations

$$
\rho^{\prime} \gamma=\rho, \quad \lambda^{\prime}=\gamma \lambda .
$$

Theorem 21.3 (ABC extension equivalence theorem). Any homomorphism $\gamma$ of one extension (21.8) of $G$ by $Q$ into another is an equivalence.

The usual (group-theoretic) proof proceeds by calculating the image and the kernel of $\gamma$; the same calculations may now be reproduced in categorical language, eventually leaving only the equivalence factor $\theta$ in the decomposition (21.1) for $\gamma$. This theorem is also of interest because the Eilenberg-Steenrod "5 Lemma" on two exact sequences of length 5 can be reduced to this theorem, also by categorical arguments.

We mention also that various alternative characterizations of freeand-direct products (such as Theorem 19.1) as well as the EilenbergSteenrod "hexagon Lemma" can be derived from the axioms of an $\mathrm{ABC}$-category; these derivations making heavy use of the addition of homomorphisms introduced in $\$ 19$.

These deductions indicate that most of the standard properties ${ }^{11}$ of homomorphisms between abelian groups (or, of linear transforma-

11 Facts such as the existence of infinite direct and infinite free products (no longer identical) or the existence, within the category, of the group $\operatorname{Hom}(A, B)$ of all homomorphisms of $A$ in to $B$ are of course not deducible from our axioms. 
tions between vector spaces) can be deduced from our self-dual system of axioms for an abelian bicategory. We thus provide a precise axiomatic proof for the duality phenomena in abelian groups.

\section{BIBLIOGRAPHY}

1. E. Artin, The free product of groups, Amer. J. Math. vol. 69 (1947) pp. 1-4.

2. P. Bernays, $A$ system of axiomatic set theory, Journal of Symbolic Logic, I, vol. 2 (1937) pp. 65-77; II, vol. 6 (1941) pp. 1-17; III, vol. 7 (1942) pp. 65-89; IV, vol. 7 (1942) pp. 133-145; V, vol. 8 (1943) pp. 89-106; VI, vol. 13 (1948) pp. 65-79.

3. G. Birkhoff, On the structure of abstract algebras, Proc. Cambridge Philos. Soc. vol. 31 (1935) pp. 433-454.

4. - Universal algebra, Proceedings of the First Canadian Mathematical Congress, Montreal, 1945, pp. 310-326.

5. —, Lattice theory, Amer. Math. Soc. Colloquium Publications vol. 25, rev. ed., New York, 1948.

6. N. Bourbaki, Éléments de mathematique, vol. II, Algèbre, Chap. I. Structures Algébriques, Paris, 1946.

7. S. Eilenberg and S. MacLane, Group extensions and homology, Ann. of Math. vol. 43 (1942) pp. 757-831.

8. - General theory of natural equivalences, Trans. Amer Math. Soc. vol. 58 (1945) pp. 231-295.

9. S. Eilenberg and N. E. Steenrod, Axiomatic approach to homology theory, Proc. Nat. Acad. Sci. U. S. A. vol. 31 (1945) pp. 117-120.

10. - Foundations of algebraic topology, to appear.

11. K. Gödel, The consistency of the continuum hypothesis, Annals of Mathematics Studies, no. 3, Princeton, 1940.

12. P. Hall, A contribution to the theory of groups of prime power order, Proc. London Math. Soc. (2) vol. 36 (1933) pp. 29-95.

13. - Verbal and marginal subgroups, Journal für Mathematik vol. 182 (1940) pp. 156-157.

14. W. Hurewicz, $Z u$ einer Arbeit von O. Schreier, Abh. Math. Sem. Hamburgischen Univ. vol. 8 (1930) pp. 307-314.

15. B. Jónsson and A. Tarski, Direct decomposition of finite algebraic systems, Notre Dame Mathematical Lectures, no. 5, Notre Dame, 1947.

16. S. MacLane, Groups, categories, and duality, Proc. Nat. Acad. Sci. U.S.A. vol. 34 (1948) pp. 263-267.

17. S. MacLane and J. H. C. Whitehead, On the 3-type of a complex, Proc. Nat. Acad. Sci. U.S.A. vol. 36 (1950) pp. 41-47.

18. W. Magnus, Allgemeine Gruppentheorie, Enzykopädie der Mathematischen Wissenschaften, 2d ed., vol. I, 1, no. 4, part 1, Leipzig and Berlin, 1939.

19. K. Shoda, Ueber die allgemeinen algebraischen Systemen, Proc. Imp. Acad. Tokyo, I, vol. 17 (1941) pp. 323-327; II, III, IV, vol. 18 (1942) pp. 179-184, 227-232, 276-279; V, VI, VII, vol. 19 (1943) pp. 114-118, 259-263, 515-517; VIII, vol. 20 (1944) pp. 584-588.

20. A. Tarski, Cardinal algebras, Oxford and New York, 1949.

21. B. L. van der Waerden, Free products of groups, Amer. J. Math. vol. 70 (1948) pp. 527-528.

22. H. Zassenhaus, Lehrbuch der Gruppentheorie, Leipzig and Berlin, 1937.

The University of Chicago 